\title{
Oxygen and hydrogen peroxide reduction by 1,2-diferrocenylethane at a liquid/liquid interface
}

\author{
Haiqiang Deng ${ }^{a}$, Pekka Peljo ${ }^{\mathrm{b}}$, Fernando Cortés-Salazar ${ }^{a}$, Peiyu Ge ${ }^{\mathrm{a}}$, Kyösti Kontturi ${ }^{\mathrm{b}}$, \\ Hubert H. Girault ${ }^{\mathrm{a}, *}$ \\ a Laboratoire d'Electrochimie Physique et Analytique, Ecole Polytechnique Fédérale de Lausanne (EPFL), Station 6, CH-1015 Lausanne, Switzerland \\ ${ }^{\mathrm{b}}$ Department of Chemistry, Aalto University, P.O. Box 16100, 00076 Aalto, Finland
}

\section{A R T I C L E I N F O}

\section{Article history:}

Received 8 February 2012

Received in revised form 11 April 2012

Accepted 14 April 2012

Available online 7 June 2012

\section{Keywords:}

ITIES

Oxygen reduction

Hydrogen peroxide reduction

1,2-Diferrocenylethane

\begin{abstract}
A B S T R A C T
Molecular oxygen and hydrogen peroxide reduction by 1,2-diferrocenylethane (DFcE) was investigated at a polarized water/1,2-dichloroethane (W/DCE) interface. The overall reaction points to a proton-coupled electron transfer (PCET) mechanism, where the first step consists of the protonation of DFcE to form the $\mathrm{DFCE}-\mathrm{H}^{+}$in DCE phase, either by DFcE facilitated proton transfer across the liquid-liquid interface or by the homogeneous protonation of DFCE in the presence of protons extracted in the oil phase by tetrakis(pentafluorophenyl)borate. The formation of $\mathrm{DFcE}-\mathrm{H}^{+}$is followed up by the $\mathrm{O}_{2}$ reduction to hydrogen peroxide and further reduction to water. The final products of $\mathrm{DFcE}$ oxidation, namely $\mathrm{DFcE}^{+}$or $\mathrm{DFcE}^{2+}$, were investigated by ion transfer voltammetry, ultramicroelectrode voltammetry and UV/visible spectroscopy. These results show that mostly $\mathrm{DFcE}^{+}$is produced, although $\mathrm{DFcE}^{+}$can also reduce oxygen at longer time scales. Hydrogen peroxide reduction is actually faster than oxygen reduction, but both reactions are slow due to relatively low thermodynamic driving force.
\end{abstract}

(c) 2012 Elsevier B.V. All rights reserved.

\section{Introduction}

The charge transfer processes across the interface between two immiscible electrolyte solutions (ITIES) are of fundamental importance for a variety of applications such as in storage and conversion of energy, solvent extraction, electroanalysis, and life sciences [1]. Within the context of green energy, vital processes such as photosynthesis and respiration (i.e. oxygen reduction) taking place at the lipid bilayers of biomembranes can be studied at the ITIES. Oxygen reduction reaction (ORR) at the water/1,2-dichloroethane interface (W/DCE) has been studied for more than 10 years, since Kihara and co-workers showed that tetrachlorohydroquinone in oil phase could reduce oxygen to water or hydrogen peroxide, depending on the potential difference applied at the W/DCE [2]. This work was one of the earliest studies of a PCET reaction at the ITIES as the reduction of oxygen in DCE requires a suitable electron donor (D) in the oil phase and a proton source in the aqueous phase. In recent years, we have also investigated the oxygen reduction at the ITIES by direct electron donors such as different ferrocene (Fc) derivatives (for example decamethylferrocene (DMFc)) $[3,4]$ or tetrathiafulvalene (TTF) [5]. Electrocatalysis of oxygen reduction by different porphyrins [6-11] and dodecylaniline [12] has also been studied, and Peljo et al. demonstrated a novel fuel cell based

\footnotetext{
* Corresponding author. Tel.: +41 21693 3145; fax: +41 216933667.

E-mail address: hubert.girault@epfl.ch (H.H. Girault).
}

on molecular catalysis of oxygen reduction at a liquid/liquid interface [13]. More recently, Olaya et al. observed the direct fourelectron reduction of oxygen at the ITIES catalyzed by self-assembled molecular rafts formed of two oppositely charged water-soluble porphyrins [14] and Peljo et al. investigated the mechanism of oxygen reduction by so-called cofacial "Pacman" type porphyrins at the ITIES [15]. The biphasic system aforementioned appears superior over the extensively-investigated homogeneous system since on the one hand, the ITIES provides a physical separation of the reactants and products (water and hydrogen peroxide are transferred back to aqueous phase) resulting in easier product collection and higher yields for systems at equilibrium (according to Le Chatelier's principle, the equilibrium can be shifted on the favor of products by extraction of products [16]); on the other hand, the reaction rate is controlled by the proton concentration in the oil phase, which is determined by the Galvani potential difference across the interface that is conveniently controlled by modern electrochemical techniques.

ORR by metallocenes at liquid/liquid interface has been proposed to proceed in two steps: proton transfer from the aqueous to the oil phase facilitated by the metallocene followed by a homogenous oxygen reduction in the oil phase. In the case of DMFc a formation of the hydride $\mathrm{DMFcH}^{+}$with the proton binding to the iron is the first step [17]. Density functional theory (DFT) calculations suggest that instead of the coordination of a triplet molecular oxygen to the iron atom (spin-forbidden) [18] or insertion into Fe$\mathrm{H}$ bond, the reaction with oxygen proceeds through a delocalized 
triplet transition state, leading to the formation of $\mathrm{DMFc}^{+}$and a hydrogen peroxyl radical [17]. Also, a mechanism where molecular oxygen is coordinated between two protonated ferrocenes has been proposed [19]. This mechanism has some similarities with the oxygen reduction by cofacial metal pophyrins [20,21], mimicking the oxygen reduction occurring in the bimetallic iron/copper center of cytochrome $c$ oxidase [22]. Because of this we decided to study oxygen reduction by 1,2-diferrocenylethane, a multiferrocenyl compound, at the polarized water/DCE interface. This compound has been successfully used as an electron donor for electron transfer studies at the liquid/liquid interface [23,24], and previous NMR results indicate that protonation of both ferrocenyl groups should take place in boron trifluoride monohydrate solution [25]. Thus, ORR to hydrogen peroxide could take place with molecular oxygen sandwiched between the protonated centers. The experimental results show in fact, that two DFcE molecules are needed for two-electron oxygen reduction, and thus cast doubt on whether diprotonated DFcE is indeed formed in DCE. Hydrogen peroxide reduction is less well understood, as this reaction is mentioned only to explain observed four-electron oxygen reduction $[18,26]$. A mechanism suggested by Fomin indicates that the protonated ferrocene can react with $\mathrm{H}_{2} \mathrm{O}_{2}$ forming water, $\mathrm{Fc}^{+}$and $\mathrm{OH}^{*}$ radical, which further reacts with $\mathrm{Fc}$ and a proton to produce water [19]. From this point of view, DFcE seems ideal for hydrogen peroxide reduction, as hydrogen peroxide can react with one protonated ferrocenyl group and then the generated $\mathrm{OH}^{*}$ radical can easily oxidize the other ferrocenyl group. The experimental results obtained in this work show that hydrogen peroxide reduction is faster than oxygen reduction, corroborating the proposed mechanism.

\section{Experimental section}

\subsection{Chemicals}

All chemicals are analytical grade and used as received without further purification. 1,2-diferrocenylethane (DFcE) was purchased from Aldrich. Anhydrous lithium chloride ( $\mathrm{LiCl}$ ), bis(triphenylphosphoranylidene) ammonium chloride (BACl), lithium sulfate $\left(\mathrm{Li}_{2} \mathrm{SO}_{4}\right), 1,2$-dichloroethane (DCE), sodium iodide (NaI), and tetramethylammonium sulfate $\left(\mathrm{TMA}_{2} \mathrm{SO}_{4}\right)$ were obtained from Fluka. Lithium tetrakis(pentafluorophenyl)borate diethyl etherate (LiTB) was purchased from Boulder Scientific and sulfuric acid $\left(\mathrm{H}_{2} \mathrm{SO}_{4}, 95-97 \%\right)$ was purchased from Sigma-Aldrich. Potassium bis(oxalato)-oxotitanate(IV) dihydrate was provided by Alfa Aesar. Bis(triphenylphosphoranylidene)ammonium tetrakis(pentafluorophenyl)borate (BATB) was prepared by metathesis of $1: 1$ mixtures of $\mathrm{BACl}$ and LiTB, in a methanol/water $(\mathrm{v} / \mathrm{v}=2)$ mixture, followed by recrystallization in acetone. The aqueous solutions were prepared with ultrapure water $(18.2 \mathrm{M} \Omega \mathrm{cm})$ from a Millipore-Q system.

\subsection{Two-phase reactions controlled by a common ion distribution (shake flask reactions)}

Two-phase shake flask reactions for oxygen reduction were performed in a small flask under stirring. For these experiments, equal volumes $(2 \mathrm{~mL})$ of DCE and aqueous solutions containing the reactants (composition of both phases shown in Scheme 1) were mixed together and stirred vigorously. After reaction aqueous and organic phases were separated and the UV-Vis spectrum of the DCE phase was measured directly. The aqueous phase was treated with excess $\mathrm{NaI}$ (equivalent to $0.1 \mathrm{M}$ ). Hydrogen peroxide reacted with $\mathrm{I}^{-}$to produce $\mathrm{I}_{3}^{-}$, which has an absorbance at $352 \mathrm{~nm}$ [4]. UV/visible (UV/Vis) spectra were obtained with an Ocean Optics CHEM2000 spectrophotometer with a quartz cuvette (path length: $10 \mathrm{~mm}$ ).

\section{\begin{tabular}{l|l}
$5 \mathrm{mM}$ BATB & $\begin{array}{l}5 \mathrm{mM} \mathrm{LiTB} \\
5 \mathrm{mM} \text { DFCE }\end{array}$
\end{tabular} DCE Water}

Scheme 1. Schematic representation of the initial compositions of the aqueous phase and the organic phase for studying oxygen reduction in a biphasic reaction.

To confirm the production of hydrogen peroxide, also the titanium oxalate method reported by Sellers was used [27]. Briefly, $1 \mathrm{~mL}$ sample of aqueous phase was acidified with sulfuric acid and mixed with potassium bis(oxalato)-oxotitanate(IV) solution to form a yellow complex with hydrogen peroxide. For quantitative purposes, the absorption of the complex was measured at $400 \mathrm{~nm}$ [27].

To study the amount of DFcE consumed in the reaction, the ratio of different DFcE species was determined by measuring cyclic voltammograms (CVs) at a scan rate of $20 \mathrm{mV} \mathrm{s}^{-1}$ with a Pt $(25 \mu \mathrm{m}$ diameter), a carbon fiber ( $10 \mu \mathrm{m}$ diameter) and a glassy carbon $(10 \mu \mathrm{m}$ diameter, Princeton Applied Research) ultramicroelectrodes (UMEs) with a CHI900 electrochemical workstation $(\mathrm{CH}$ Instruments, Austin, USA). For comparison, CVs of a freshly prepared DCE solution of $5 \mathrm{mM}$ DFCE under anaerobic conditions were also recorded. For achieving the anaerobic conditions the solution was degassed by bubbling pure $\mathrm{N}_{2}$ through it for $30 \mathrm{~min}$ and then keeping a $\mathrm{N}_{2}$ atmosphere over the solution during the voltammetric measurements. For recording the CVs, a three-electrode system with a Pt wire as the counter electrode and a $\mathrm{Ag} / \mathrm{AgTB}$ wire as the reference electrode (diameter $=0.5 \mathrm{~mm}$, made by electrolysis of a $\mathrm{Ag}$ wire in $10 \mathrm{mM}$ LiTB solution) was employed. The potential scale was calibrated with the addition of decamethylferrocene $(0.04 \mathrm{~V}$ vs. SHE in DCE [28]) at the end of the voltammetry experiments.

Fabrication of the Pt and carbon fiber UMEs was performed by sealing a $25 \mu \mathrm{m}$-diameter Pt wire or a $10 \mu \mathrm{m}$-diameter carbon fiber (Goodfellow, Oxford, UK) at one end of a glass capillary (i.e. inner diameter $1 \mathrm{~mm}$, outer diameter $1.5 \mathrm{~mm}$, Bio-logic) by a butane/propane/oxygen flame (C206 Super, CAMPINGAZ). Afterwards, the glass capillary with a sealed Pt wire or carbon fiber is subject to a vacuum system for c.a. $30 \mathrm{~min}$. Then, the capillary is slowly sealed onto Pt wire or the carbon fiber by placing it inside a resistor heater coil (Model 720, David Kopf Instruments, USA). Special attention has to be paid in order to place the capillary in the center of the coil to avoid capillary deformations leading to non-straight UMEs. After a capillary section equal to $1 \mathrm{~cm}$-length is properly sealed, the electrical connection is made by melting a given amount of tin powder between the Pt wire or carbon fiber and a larger tin/copper lead wire. Lastly, the tin/copper lead wire is fixed to the glass capillary by a two-component epoxy resin (Araldit, Reckitt \& Colman AG), letting a free tin/copper wire section outside the glass capillary for electrical connection purposes [29].

Hydrogen peroxide decomposition was investigated by having $\mathrm{H}_{2} \mathrm{O}_{2}$ solution in contact with DFcE in the DCE phase (Scheme 2), without a partition of a common ion. The reaction was monitored with UV/Vis spectroscopy and UME voltammetry.

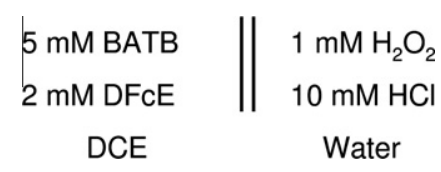

Scheme 2. Schematic representation of the initial composition of the aqueous phase and the organic phase for studying hydrogen peroxide decomposition in a biphasic reaction. 


\begin{tabular}{|c|c|}
\hline 5 mM BATB & $1 \mathrm{mM} \mathrm{H}_{2} \mathrm{O}_{2}$ \\
\hline \multirow[t]{2}{*}{$2.6 \mathrm{mM}$ DFcE } & 5 mM LiTB \\
\hline & $10 \mathrm{mM} \mathrm{HCl}$ \\
\hline DCE & Water \\
\hline
\end{tabular}

Scheme 3. Schematic representation of the initial composition of the aqueous phase and the organic phase for studying hydrogen peroxide reduction in a biphasic reaction.

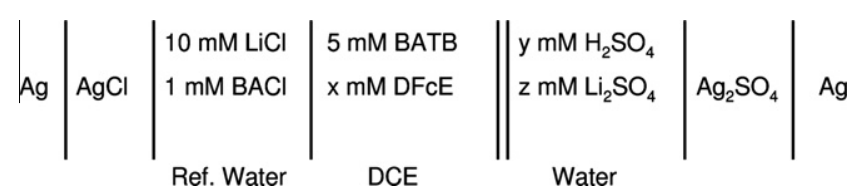

Scheme 4. Schematic depiction of the electrochemical cell composition used in the four-electrode configuration.

Hydrogen peroxide reduction was studied in a glove box with a nitrogen atmosphere, using the cell described in Scheme 3. For this, equal volumes of deoxygenated solutions were mixed together and stirred vigorously for $30 \mathrm{~min}$, and the reaction products were analyzed as described above, with UV/Vis spectroscopy and UME voltammetry with a glassy carbon electrode.

\subsection{Electrochemical measurements}

All the electrochemical measurements were performed at ambient temperature $\left(20 \pm 2^{\circ} \mathrm{C}\right)$ under aerobic conditions in a Faraday cage. CVs at the W/DCE interface were obtained using an Autolab four-electrode potentiostat (PGSTAT 30, Eco-chemie, the Netherlands). Two glass cells designed for liquid-liquid interface experiments with the interfacial area of $0.159 \mathrm{~cm}^{2}$ (a generous gift from prof. Zdeněk Samec, J. Heyrovský Institute of Physical Chemistry, Prague) or $1.53 \mathrm{~cm}^{2}$ were used in the experiments. Two reference electrodes $\left(\mathrm{Ag} / \mathrm{Ag}_{2} \mathrm{SO}_{4}\right.$ or $\mathrm{Ag}$ quasi-reference electrode (AgQRE), and $\mathrm{Ag} / \mathrm{AgCl}$ ) were placed in Luggin capillaries to reduce the $i R$ drop, and used for controlling the potential difference across the interface, while tungsten or platinum counter electrodes in both phases provided the current. The organic reference phase had a common cation with the supporting electrolyte of the organic phase, as described in Scheme 4 . The potential was converted to the Galvani potential scale $\left(\Delta_{\mathrm{o}}^{\mathrm{w}} \phi\right)$, based on CV measurement of the reversible half-wave potential $\Delta_{\mathrm{o}}^{\mathrm{w}} \phi_{\mathrm{TMA}^{+}}^{1 / 2}$ of the $\mathrm{TMA}^{+}$ion transfer (0.16 V in DCE [30]).

\section{Results and discussion}

\subsection{Redox properties of DFCE}

The redox properties of DFcE in DCE were studied by cyclic voltammetry as shown in Fig. 1. From this figure, it can be seen that $\mathrm{DFCE}$ has two oxidation waves corresponding to $\mathrm{DFCE}^{+} / \mathrm{DFcE}$ and $\mathrm{DFcE}^{2+} / \mathrm{DFcE}^{+}$in DCE with the half-wave potentials $E_{1 / 2}$ at $0.565 \mathrm{~V}$ and $0.770 \mathrm{~V} v$ s. SHE (i.e. $E_{1 / 2}$ separation $205 \mathrm{mV}$ ), respectively. It has been reported previously for DFcE in dichloromethane a $E_{1 / 2}$ separation of $180 \mathrm{mV}$, when $\mathrm{TB}^{-}$was used as a counter anion [31]. The fact that two consecutive oxidation waves (i.e. one electron transfer for each wave) are observed for DFcE in cyclic voltammetry instead of a single two-electron transfer process, indicates that strong intramolecular electronic communication between the two ferrocenyl groups in the same reactant molecule occurred [32]. When one of the two ferrocenyl groups is oxidized, the

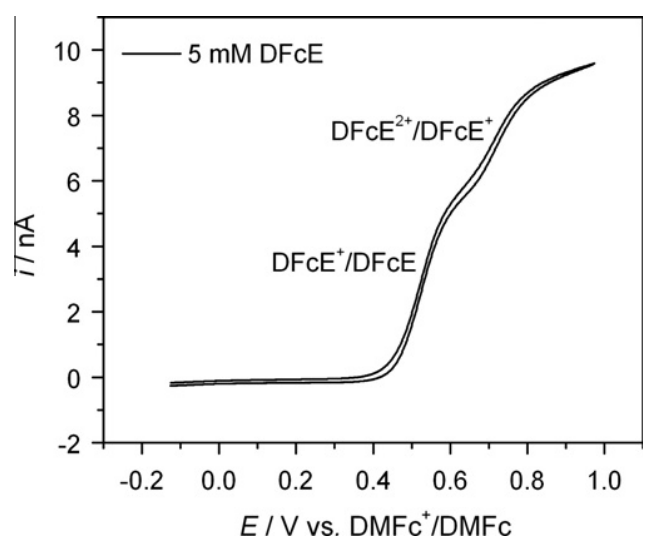

Fig. 1. Cyclic voltammogram $\left(20 \mathrm{mV} \mathrm{s}^{-1}\right)$ of freshly prepared $5 \mathrm{mM}$ DFcE solution under $\mathrm{N}_{2}$ atmosphere on a carbon fiber UME (diameter $=10 \mu \mathrm{m}$ ). The potential scale was referred to the $\mathrm{DMFc}^{+} / \mathrm{DMFc}$ couple.

electron-withdrawing monocation $\mathrm{DFcE}^{+}$is formed, which leads to the oxidation of the second ferrocenyl group to the dication, $\mathrm{DFCE}^{2+}$, at a more positive potential. Accordingly, the strong electrostatic interaction between the positively charged ferrocenyl groups (via stepwise oxidation) results in significant wave separation as shown in Fig. 1 [33]. The latter is further explained by the effect that the employed solvent and supporting electrolyte have on the separation of the two oxidation waves. Generally more strongly coordinating anions like $\mathrm{ClO}_{4}^{-}, \mathrm{BF}_{4}^{-}$and $\mathrm{PF}_{6}^{-}$are able to stabilize the formed monocations by ion-pairing, offsetting the electron-withdrawing effect of $\mathrm{DFcE}^{+}$toward the other ferrocenyl group and decreasing the difference between the observed halfwave potentials of the two oxidation waves. Contrarily, the use of weakly coordinating anions like $\mathrm{TB}^{-}$increases the separation of the observed half-wave potentials. The solvent has a similar effect: highly polar solvents like dimethyl sulfoxide and solvents with significant donor character (for example tetrahydrofuran, donor number $=20$ ) are also able to stabilize the formed monocations, making the second oxidation easier to take place [34]. For example, in a polar solvent like dimethylformamide, DFcE has only one oxidation wave when $\mathrm{ClO}_{4}^{-}$is used as a counter anion [35].

Voltammetry measurements show that the formal potential of DFcE species ( 0.565 and $0.770 \mathrm{~V} v$ s. SHE) is not low enough for proton reduction to occur in the oil phase (standard redox potential of $0.55 \mathrm{~V} v s$. SHE in DCE [36]), but oxygen reduction in the presence of protons (standard redox potential of $1.17 \mathrm{~V} v s$. SHE for hydrogen peroxide and $1.75 \mathrm{~V} v$ s. SHE for water in DCE [6]) by DFcE and $\mathrm{DFCE}^{+}$ is thermodynamically feasible. However, the reduction of oxygen to the superoxide is highly unfavorable (standard redox potential of $-0.81 \mathrm{~V}$ vs. SHE in DCE [12]). This point will be detailed in Section 3.2.1. The limiting currents measured on a $10 \mu \mathrm{m}$ carbon fiber UME were used to evaluate the diffusion coefficients of the neutral and $\mathrm{DFcE}^{+}$species as 5.8 and $3.7 \times 10^{-6} \mathrm{~cm}^{2} \mathrm{~s}^{-1}$, respectively.

\subsection{Two-phase reactions controlled by a common ion distribution}

\subsubsection{Oxygen reduction}

Oxygen reduction by DFcE is described by Eqs. (1) and (2). Ferrocene derivatives also catalyze decomposition and also further reduce hydrogen peroxide, as described by Eqs. (3) and (4).

$$
\begin{aligned}
& 2 \mathrm{DFcE}(\mathrm{o})+\mathrm{O}_{2}(\mathrm{o})+2 \mathrm{H}^{+}(\mathrm{w}) \rightarrow 2 \mathrm{DFcE}^{+}(\mathrm{o})+\mathrm{H}_{2} \mathrm{O}_{2}(\mathrm{w}) \\
& \mathrm{DFcE}(\mathrm{o})+\mathrm{O}_{2}(\mathrm{o})+2 \mathrm{H}^{+}(\mathrm{w}) \rightarrow \mathrm{DFCE}^{2+}(\mathrm{o})+\mathrm{H}_{2} \mathrm{O}_{2}(\mathrm{w}) \\
& 2 \mathrm{H}_{2} \mathrm{O}_{2}(\mathrm{w}) \stackrel{\mathrm{DFCE}}{\longrightarrow} \mathrm{O}_{2}(\mathrm{w})+2 \mathrm{H}_{2} \mathrm{O}(\mathrm{w})
\end{aligned}
$$




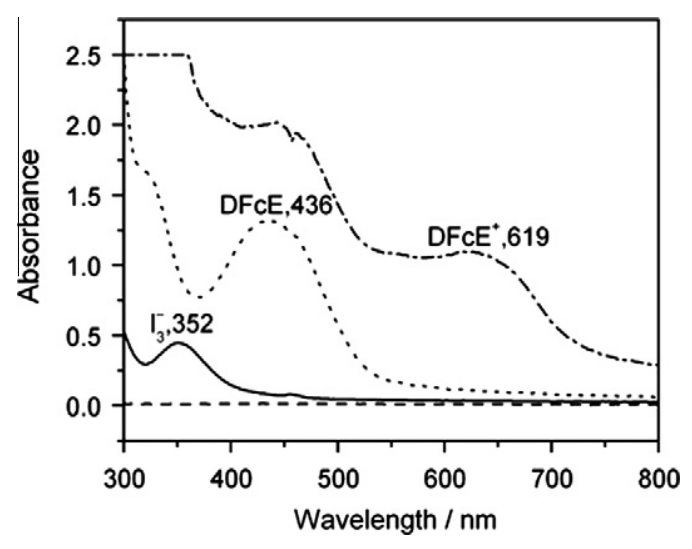

Fig. 2. UV/Vis spectra of the aqueous phase before (control, dashed line) and after (target, solid line) $10 \mathrm{~min}$ of aerobic two-phase reaction under stirring conditions: both of the aqueous solutions (control and target) were treated with $0.1 \mathrm{M} \mathrm{NaI}$ prior to UV/Vis measurements. The dotted and dash-dotted traces correspond to the UV/ Vis spectra of DFcE solutions in DCE before and after $10 \mathrm{~min}$ of aerobic two-phase reaction under stirring conditions (diluted by half), respectively. For the two-phase reaction: the aqueous phase contained $50 \mathrm{mM} \mathrm{H}_{2} \mathrm{SO}_{4}+5 \mathrm{mM}$ LiTB $(2 \mathrm{~mL})$; the DCE phase contained $5 \mathrm{mM}$ DFcE $+5 \mathrm{mM}$ BATB $(2 \mathrm{~mL})$.

$$
2 \mathrm{DFcE}(\mathrm{o})+\mathrm{H}_{2} \mathrm{O}_{2}(\mathrm{w})+2 \mathrm{H}^{+}(\mathrm{w}) \longrightarrow 2 \mathrm{DFcE}^{+}(\mathrm{o})+2 \mathrm{H}_{2} \mathrm{O}(\mathrm{w})
$$

The reduction of $\mathrm{O}_{2}$ by DFcE was investigated by shake-flask experiments, where the Galvani potential difference across the interface is controlled by a common ion distribution. When $\mathrm{TB}^{-}$ was used as a common ion, the Galvani potential difference is fixed at a potential greater than $0.59 \mathrm{~V}$, so that protons are extracted to oil phase. A fresh solution of DFcE in DCE has a brown color and displays an absorption band in the UV/Vis spectrum at $\lambda_{\max }=436 \mathrm{~nm}$ (dotted curve in Fig. 2). After the two-phase shake flask reaction (i.e. reaction time $=10 \mathrm{~min}$ ), the DCE phase turned dark green, and a broad absorption band at $\lambda_{\max }=619 \mathrm{~nm}$ (dash-dotted curve in Fig. 2) corresponding to $\mathrm{DFcE}^{+}$was observed. The presence of $\mathrm{H}_{2} \mathrm{O}_{2}$ in the aqueous solution after the shake-flask reaction was confirmed with the NaI and titanium oxalate (data not shown) methods, as represented by the appearance of the $\mathrm{I}_{3}^{-}$characteristic absorption band at $\lambda_{\max }=352 \mathrm{~nm}$ (solid curve in Fig. 2). On the contrary, no signal was observed in the UV/Vis spectrum for the aqueous phase before the biphasic reaction (dashed curve in Fig. 2).

Formation of the $\mathrm{DFcE}^{+}$cation was also confirmed by the cyclic voltammograms of a carbon fiber (diameter $=10 \mu \mathrm{m}$ ) and a Pt (diameter $=25 \mu \mathrm{m}$ ) UMEs in the isolated DCE phase after shakeflask reaction, as illustrated in Fig. 3. After $10 \mathrm{~min}$ of shake-flask reaction, three steady-state current waves, namely $\mathrm{DFcE}^{2+} \leftrightarrow \mathrm{DFcE}^{+}$, $\mathrm{DFcE}^{+} \leftrightarrow \mathrm{DFcE}$, and $\mathrm{H}^{+} \rightarrow 1 / 2 \mathrm{H}_{2}$ were observed at the $\mathrm{DMFc}^{+} / \mathrm{DMFc}$ potential scale. The percentage of DFcE oxidized to $\mathrm{DFcE}^{+}$could be calculated from the first oxidation wave of DFcE to be about $20 \%$, so ca. $1 \mathrm{mM} \mathrm{DFcE}^{+}$was generated during the reaction. The sum of the magnitudes of cathodic and anodic current for $\mathrm{DFcE}^{+} \leftrightarrow \mathrm{DFcE}$ is close to that of freshly prepared DFcE in DCE (data not shown) showing almost no $\mathrm{DFCE}^{2+}$ was produced in the time scale of this experiment. Also, the half-wave potential $E_{1 / 2}$ for $\mathrm{H}^{+}$reduction at a carbon UME is about $577 \mathrm{mV}$ more negative than that at a $\mathrm{Pt}$ UME, as observed in Fig. 3. This makes carbon UMEs more suitable for studying the products of this reaction. The selectivity for the production of hydrogen peroxide over water was less than $6 \%$, calculated based on the amount of produced hydrogen peroxide and the amount of consumed DFcE.

A shake flask experiment with LiTB:DFcE ( 5 mM DFcE) molar ratio of 4 was performed for $48 \mathrm{~h}$, and the conversion of DFcE was monitored by voltammetry with a carbon fiber UME during reaction. About half of the DFcE had been oxidized to DFcE ${ }^{+}$after $1 \mathrm{~h}$,

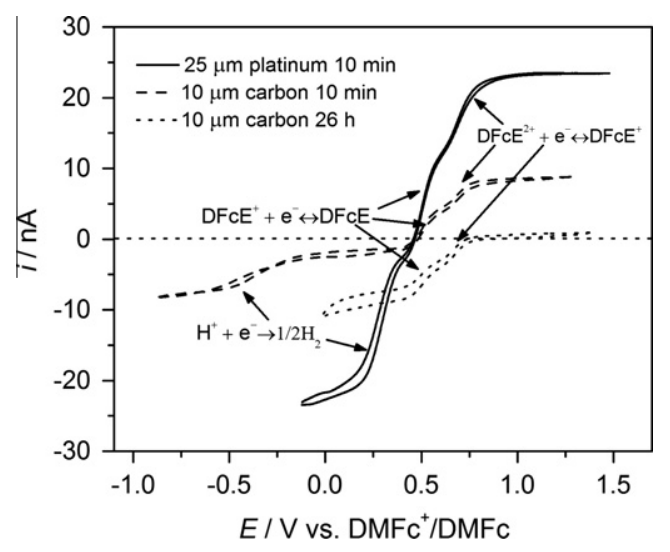

Fig. 3. Cyclic voltammograms at a Pt (diameter $=25 \mu \mathrm{m}$, solid line) and a carbon fiber UME (diameter $=10 \mu \mathrm{m}$ ) located in DCE solution containing $5 \mathrm{mM} \mathrm{DFCE}$ and $5 \mathrm{mM}$ BATB after $10 \mathrm{~min}$ (dashed line) of the two-phase shake flask reaction under aerobic conditions, in which the potential scale was referred to the DMFc ${ }^{+} / \mathrm{DMFc}$ couple. For comparison, cyclic voltammogram of $\mathrm{DFcE}^{2+} / \mathrm{DFcE}^{+}$solution obtained by $26 \mathrm{~h}$ shake flask experiment (dotted line) under aerobic conditions on a carbon fiber UME (diameter $=10 \mu \mathrm{m}$ ) is included. Scan rate is $20 \mathrm{mV} \mathrm{s}^{-1}$. The horizontal line depicts the position of zero current.

but further oxidation to $\mathrm{DFcE}^{2+}$ took much longer time. After $18 \mathrm{~h}$ about half of the DFcE was converted to $\mathrm{DFcE}^{2+}$, and the reaction was not complete until after $26 \mathrm{~h}$ of reaction (see Fig. 3 ). Results show that the kinetics of oxygen reduction by DFcE is very slow, and the produced $\mathrm{DFcE}^{+}$is also able to reduce oxygen, although at even slower rate, as expected from the higher redox potential. Ferrocene derivatives have been shown to catalyze the decomposition of hydrogen peroxide, and they may also be able to further reduce hydrogen peroxide [26]. Thus, with such a long time scale the reactions with hydrogen peroxide are not negligible, and the exact efficiency of hydrogen peroxide production cannot be evaluated from shake flask experiments. The CV of the organic phase after $26 \mathrm{~h}$ of reaction shown in Fig. 3 demonstrates that the complete oxidation of DFcE to $\mathrm{DFcE}^{2+}$ is possible. The diffusion coefficient of $\mathrm{DFcE}^{2+}$ calculated from the first steady-state cathodic process observed with this solution (dotted line in Fig. 3 ) is equal to $3.8 \times 10^{-6} \mathrm{~cm}^{2} \mathrm{~s}^{-1}$, almost the same as that of $\mathrm{DFCE}^{+}$.

A control shake-flask experiment without aqueous acid (Scheme 1, without aqueous $\mathrm{H}_{2} \mathrm{SO}_{4}$ ) under aerobic conditions was also conducted (reaction time $10 \mathrm{~min}$, data not shown). The color of DCE phase turned slightly green after the two-phase shake flask reaction, while the characteristic absorption peaks both for $\mathrm{DFcE}^{+}$in $\mathrm{DCE}$ phase and that for $\mathrm{I}_{3}^{-}$in aqueous are not evident, indicating that the reaction is even slower in the absence of aqueous additional acid. The CV recorded with a glassy carbon UME (10 $\mu$ m diameter) showed that ca. $9.7 \%$ of DFcE was oxidized, while this value was about $20 \%$ with $50 \mathrm{mM} \mathrm{H}_{2} \mathrm{SO}_{4}$ in the aqueous phase. The $\mathrm{pH}$ in aqueous phase increased from initial value of 7.72 ( $5 \mathrm{mM}$ LiTB) to 9.68 during the experiment. The reaction can be described as the oxygen reduction in alkaline conditions (Eq. (5)). From the experimental point of view, it is not easy to distinguish between the reaction going through the superoxide and the one producing $\mathrm{HO}_{2}^{-}$by a single direct reaction, but thermodynamically superoxide step is highly unfavorable, and thus less likely than the $\mathrm{HO}_{2}^{-}$pathway. The protons present in the aqueous phase are extracted into the oil phase by $\mathrm{TB}^{-}$and more water will dissociate to protons and $\mathrm{OH}^{-}$to keep the system in equilibrium. As the concentration of protons at aqueous $\mathrm{pH}>7$ is very small, this extraction is slow. Protons react with oxygen and DFcE in the oil phase, and hence more protons are extracted to keep the distribution of ions between both phases at equilibrium. The concentration of $\mathrm{OH}^{-}$increases in the aqueous phase, increasing the $\mathrm{pH}$. 
$2 \mathrm{DFcE}+\mathrm{O}_{2}+\mathrm{H}_{2} \mathrm{O} \rightarrow \mathrm{HO}_{2}^{-}+\mathrm{OH}^{-}+2 \mathrm{DFcE}^{+}$

It should be noted that under aqueous acidified conditions, reaction in Eq. (5) will be the same as described in Eq. (1).

\subsubsection{Hydrogen peroxide decomposition and reduction}

The effect of hydrogen peroxide decomposition on the observed selectivity for two-electron oxygen reduction was studied by mixing equal volumes of DCE solution containing DFcE and aqueous hydrogen peroxide solution in a vial (Scheme 2). The amount of hydrogen peroxide determined with TiOx method decreased by $5 \%$ after $10 \mathrm{~min}$ and $76 \%$ after $60 \mathrm{~min}$ (in separate experiments). The aqueous phase turned slightly bluish after 60 min experiment, indicating that DFcE partitions into the aqueous phase and reduces hydrogen peroxide to form $\mathrm{DFCE}^{+}$. No oxidation of $\mathrm{DFCE}$ in the DCE phase was observed, but the amount of DFCE in DCE decreased significantly, $4 \%$ after $10 \mathrm{~min}$ and $30 \%$ after $60 \mathrm{~min}$, confirming that DFcE partitions into the aqueous phase where it reduces hydrogen peroxide. Partition coefficient ( $\log P$ ) of DFcE between DCE and water was determined to be 3.7-3.9 (see Section 3.3), so the equilibrium concentration of DFcE in water is $<0.4 \mu \mathrm{M}$. Thus, $30 \%$ of DFcE lost from the DCE phase $(0.6 \mathrm{mM})$ after $60 \mathrm{~min}$ of reaction is oxidized to $\mathrm{DFcE}^{+}$by stoichiometric amount of $0.3 \mathrm{mM}$ of hydrogen peroxide. This accounts for $40 \%$ of the loss of $\mathrm{H}_{2} \mathrm{O}_{2}$, and the rest is lost by decomposition. Hydrogen peroxide is also slightly partitioning into the DCE phase, but further reduction is now impossible as no protons are available in the DCE phase.

Hydrogen peroxide reduction was investigated in the absence of oxygen, in a glove box. $2 \mathrm{~mL}$ of DCE solution was mixed with $2 \mathrm{~mL}$ of hydrogen peroxide solution and stirred vigorously for $30 \mathrm{~min}$ (Scheme 3). The oil phase was analyzed with UME voltammetry and UV/Vis spectroscopy before and after the reaction, and the hydrogen peroxide was analyzed with TiOx and NaI - methods. Now $45 \%$ of DFcE (1.2 mM) was oxidized to $\mathrm{DFcE}^{+}$, and $\mathrm{DFcE}^{+}$peak was clearly observed in UV/Vis spectra of the DCE phase (Figs. 4 and 5 ). $96 \%$ of hydrogen peroxide was consumed (93\% when analyzed with TiOx method) during the reaction. As the stoichiometric amount of reduced hydrogen peroxide would be $0.6 \mathrm{mM}$ or $60 \%$ (Eq. (4)), part of the hydrogen peroxide was decomposed during the reaction (Eq. (3)). Now this reaction can take place in both phases, as protons and $\mathrm{DFCE}(<0.4 \mu \mathrm{M}$ in aqueous phase $)$ are both available everywhere in the system. However, the reaction is thermodynamically more favorable in the oil phase (standard redox potential of $2.31 \mathrm{~V} v$ s. SHE in DCE and $1.76 \mathrm{~V} v$ s. SHE in water [12]), and proceeds much faster than observed in the absence of LiTB $\left(60 \%\right.$ of $\mathrm{H}_{2} \mathrm{O}_{2}$ reduced after $30 \mathrm{~min} v$ s. $30 \%$ of $\mathrm{H}_{2} \mathrm{O}_{2}$ reduced

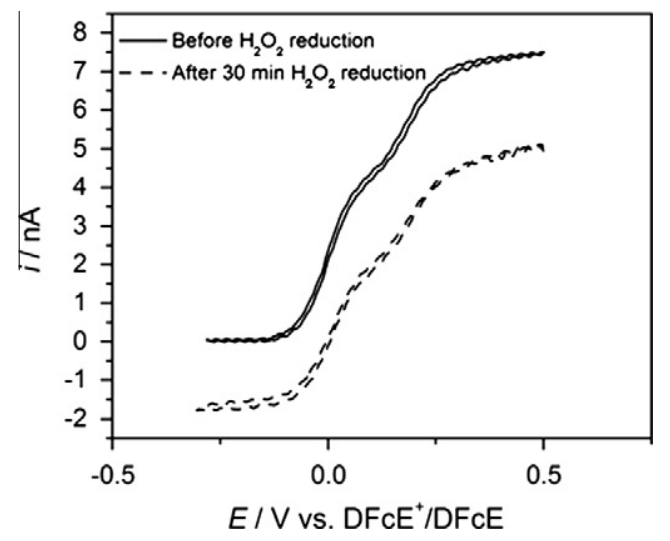

Fig. 4. Cyclic voltammograms (with $10 \mu \mathrm{m}$ diameter glassy carbon UME) of $2.6 \mathrm{mM}$ $\mathrm{DFCE}+5 \mathrm{mM}$ BATB in DCE before (solid line) and after (dashed line) 30 min biphasic hydrogen peroxide reduction inside a glove box. The potential scale was calibrated with respect to the $\mathrm{DFCE}^{+} / \mathrm{DFcE}$ couple.

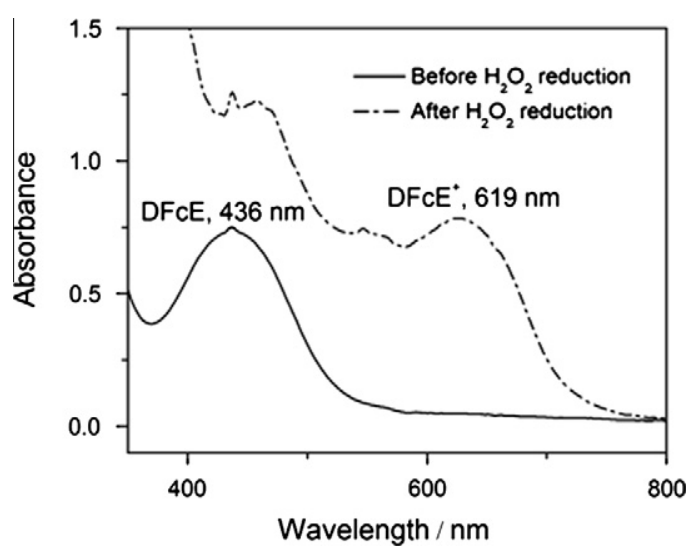

Fig. 5. UV/Vis spectra of $2.6 \mathrm{mM} \mathrm{DFcE}+5 \mathrm{mM}$ BATB in DCE before (solid line) and after (dash-dotted line) 30 min biphasic hydrogen peroxide reduction inside a glove box.

after $60 \mathrm{~min}$ in the absence of LiTB). In comparison, 60 min aerobic shake flask reaction with $2 \mathrm{mM} \mathrm{DFcE}+5 \mathrm{mM}$ BATB and $5 \mathrm{mM}$ $\mathrm{LiTB}+10 \mathrm{mM} \mathrm{HCl}$ had only a $33 \%$ conversion of $\mathrm{DFcE}$ to $\mathrm{DFcE}^{+}$, indicating that hydrogen peroxide reduction by DFcE is faster than oxygen reduction. This explains why so small amounts of hydrogen peroxide are detected in the shake flask experiments, as oxygen is firstly reduced to hydrogen peroxide, followed by faster hydrogen peroxide reduction step.

Ferrocene and its derivatives have been used as electron donors for oxygen reduction in the absence [26] or presence of catalysts [37-39], but these results show that certain care has to be taken when determining the selectivity of oxygen reduction to water, as the electron donor itself can reduce hydrogen peroxide, thus increasing the observed apparent selectivity of the catalyst towards four-electron reduction of molecular oxygen. This ability to reduce hydrogen peroxide is expected to be even stronger in the case of stronger reductants like decamethylferrocene, so control experiments both for hydrogen peroxide reduction and decomposition should always be performed.

\subsection{Four electrode cell measurements}

The CVs obtained with the four-electrode electrochemical cell described in Scheme 4 are shown in Fig. 6.

When $\mathrm{H}_{2} \mathrm{SO}_{4} / \mathrm{Li}_{2} \mathrm{SO}_{4}$ and BATB were used as the hydrophilic and hydrophobic electrolytes in aqueous and DCE phases respectively, a polarized potential window (PPW) from about -0.30 to $0.40 \mathrm{~V}$ in the Galvani potential scale at aqueous $\mathrm{pH}=1$ can be obtained, as displayed by the cyclic voltammogram shown in Fig. 6 (dotted line). The potential window is limited by the transfer of $\mathrm{H}^{+}$and $\mathrm{SO}_{4}^{2-}$ from water to DCE at positive and negative potentials (i.e. water $v s$. DCE), respectively. From a mechanistic point of view, it has been proposed that the first step in the oxygen reduction at the ITIES is the protonation of the electron donor. This is observed as a facilitated proton transfer from aqueous to oil phase by the electron donor acting as a ligand (L). The Nernst equation for this facilitated proton transfer process can be described as shown in the following equation [40]:

$$
\begin{aligned}
\Delta_{\mathrm{o}}^{\mathrm{w}} \phi_{\mathrm{LH}^{+}}^{1 / 2}= & \Delta_{\mathrm{o}}^{\mathrm{w}} \phi_{\mathrm{H}^{+}}^{0^{\prime}}+\frac{R T}{2 F} \ln \left(\frac{D_{\mathrm{L}}}{D_{\mathrm{LH}^{+}}}\right)-\frac{2.303 R T}{F} \mathrm{p} K_{a} \\
& +\frac{2.303 R T}{F} \mathrm{pH}^{\mathrm{w}}
\end{aligned}
$$

where $\Delta_{\mathrm{o}}^{\mathrm{w}} \phi_{\mathrm{LH}^{+}}^{1 / 2}$ and $\Delta_{\mathrm{o}}^{\mathrm{w}} \phi_{\mathrm{H}^{+}}^{0,}$ are the formal transfer potentials of $\mathrm{LH}^{+}$ and $\mathrm{H}^{+}(0.55 \mathrm{~V}$ at the $\mathrm{W} / \mathrm{DCE}$ interface $)$, respectively. $\mathrm{LH}^{+}$stands for the protonated complex, $D$ represents the diffusion coefficient 


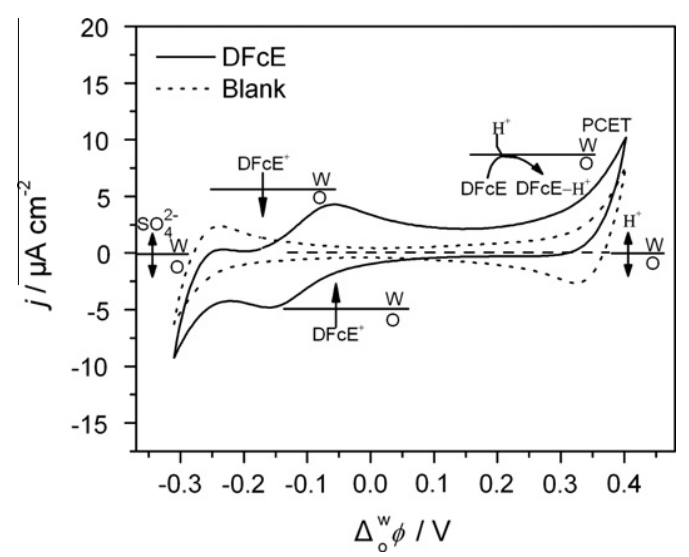

Fig. 6. Cyclic voltammograms obtained with the electrochemical cell shown in Scheme 4 in the absence of DFcE (dotted line, aerobic, $x=0, y=50, z=10$ ) and in the presence of DFcE (solid line, aerobic, $x=5, y=50, z=10$ ) in the DCE phase; scan rate: $50 \mathrm{mV} \mathrm{s}^{-1}$.

of species in the oil phase, $K_{\mathrm{a}}$ is the equilibrium constant of the protonation reaction of $\mathrm{L}$ and $\mathrm{pH}^{\mathrm{w}}$ is the aqueous $\mathrm{pH}$. Hence the ITIES essentially functions as a proton pump. The next step is the reduction of oxygen by the formed protonated complex homogeneously to produce hydrogen peroxide or water.

Under aerobic conditions, the current increased remarkably on the positive limit of the potential window upon addition of $5 \mathrm{mM}$ DFcE, and no clear return peak was observed for the transferred protons (solid line in Fig. 6), indicating that they were consumed in a homogeneous reaction according to Eqs. (1) and (2). The transfer of $\mathrm{DFCE}^{+}$generated in the oxygen reduction is observed in the range of -0.2 to $-0.05 \mathrm{~V}$ with the half-wave potential at $-0.12 \mathrm{~V}$, and the onset potential of the proton transfer took place at lower potentials, indicating the assisted proton transfer by DFcE, as described by Eq. (5). A small current resulting from proton transfer from the oil phase after reversal of the scan direction at around $0.4 \mathrm{~V}$ shows that oxygen reduction is not fast enough to consume all the transferred protons in the time scale of the experiment. Similar results have been reported previously by Su et al. when DMFc was used as electron donor for the oxygen reduction at the ITIES [4]. The forward current enhancement by DFcE is less than that by DMFc, indicating most likely slower kinetics due to the difference in electrochemical driving force $(0.04 \mathrm{~V}$ for DMFc $v$ s. $0.57 \mathrm{~V}$ vs. SHE for DFcE). These two systems are clear examples of PCET

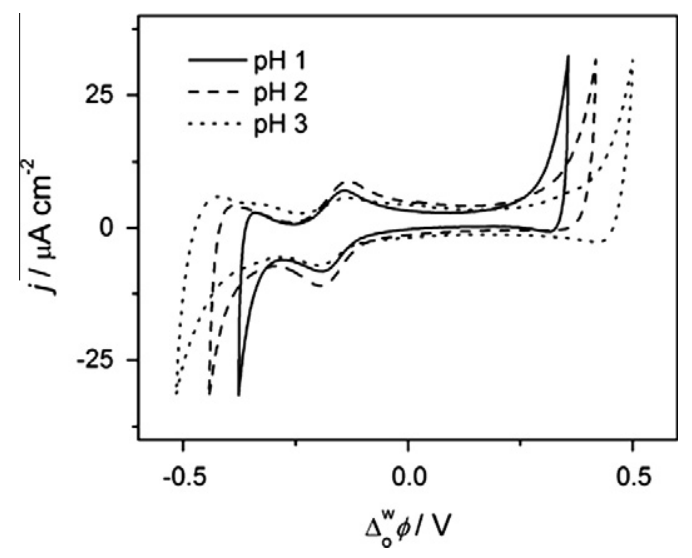

Fig. 7. Cyclic voltammogram showing the pH dependence of assisted proton transfer by DFcE under aerobic conditions. The electrochemical cell employed was shown in Scheme $4(x=5, y=50,5,0.5$ corresponding to $\mathrm{pH} 1,2,3$ of aqueous phase, respectively, $z=0)$. Scan rate $=50 \mathrm{mV} \mathrm{s}^{-1}$. reactions occurring at the ITIES. In effect, DFcE acts as a lipophilic base to complex $\mathrm{H}^{+}$during the forward potential scan, while the polarized ITIES acts as a pump to drive $\mathrm{H}^{+}$transfer from aqueous to organic phase.

Fig. 7 illustrates the $\mathrm{pH}$ dependence of the present system, in which the onset potential for assisted $\mathrm{H}^{+}$transfer shifts positively with the increasing aqueous $\mathrm{pH}$. It can be noticed that the potential window decreases as the aqueous $\mathrm{pH}$ decreases, thereby confirming that the potential window is limited by the transfer of $\mathrm{H}^{+}$and $\mathrm{SO}_{4}^{2-}$.

The scan rate dependence of the electrochemical signal observed by CV was also investigated (see Fig. 8), showing that the current magnitude for both $\mathrm{DFcE}^{+}$transfer and assisted $\mathrm{H}^{+}$transfer increases with the increase in the scan rate. Specifically, it is proportional to the square root of the scan rate (see inset in Fig. 8). This scan rate dependence indicates the overall process is limited by the linear diffusion of $\mathrm{DFcE}^{+}$(ion transfer) or DFcE (assisted proton transfer) to the interface. Besides, the CVs in Fig. 8 shows a so-called "Osakai mechanism" [41], represented by a positive current offset between 0.1 and $0.3 \mathrm{~V}$ that increases with increasing scan rate. The latter indicates that DFcE partitions between the two phases and reacts with aqueous protons and oxygen, forming $\mathrm{DFcE}^{+}$that is transferred back to oil phase at potentials higher than the observed half-wave potential for $\mathrm{DFCE}^{+}$transfer and thus producing a positive current offset in the CVs. A similar behavior has been observed for DMFc system [12]. Moreover, the increasing currents resulting from the transfer of protons from oil to aqueous phase with the scan rate during the reverse scan at $0.3-0.4 \mathrm{~V}$ indicates that the kinetics of PCET is not fast.

The uncompensated resistance broadens the observed peak of $\mathrm{DFcE}^{+}$-transfer, but if the peak potentials are plotted as a function of the scan rate and extrapolated to zero scan rate, the peak separation approaches $68 \mathrm{mV}$ (data not shown), confirming that the observed species is indeed $\mathrm{DFcE}^{+}$. Potential was scanned to even more positive potentials to see if any additional peaks from the formation of $\mathrm{DFCE}^{2+}$ or $\mathrm{DFcE}-\mathrm{H}^{+}$could be observed, but the only effects were the increase of the proton back transfer peak and the peak of $\mathrm{DFcE}^{+}$-transfer, confirming that formation of $\mathrm{DFCE}^{2+}$ is slow. This is probably due to the low proton affinity of the $\mathrm{DFcE}-\mathrm{H}^{+}$: second protonation of the molecule is not likely to take place due to the repulsion of the positive charge of $\mathrm{DFCE}-\mathrm{H}^{+}$, but instead the proton will favor association with free $\mathrm{DFcE}$ or $\mathrm{TB}^{-}$.

The $0.1 \mathrm{mM} \mathrm{DFCE}^{2+}$ DCE solution prepared by $26 \mathrm{~h}$ biphasic reaction was studied in a four electrode cell. The comparison of CVs of DFcE and DFcE ${ }^{2+}$ solutions are show in Fig. 9.

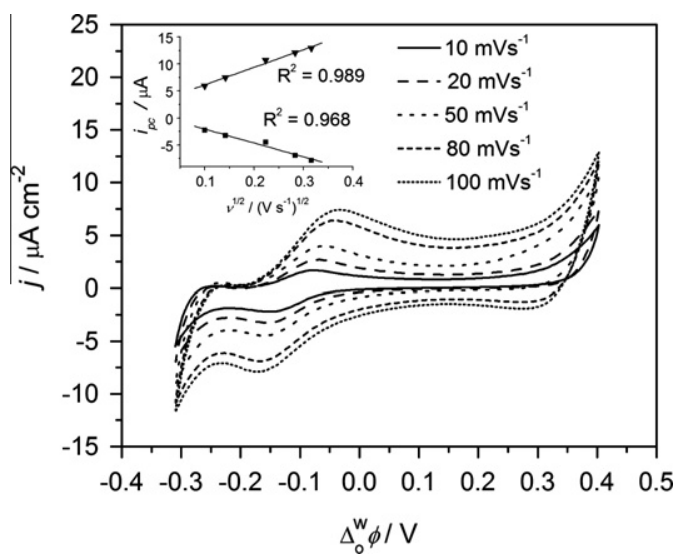

Fig. 8. Cyclic voltammograms obtained with the electrochemical cell shown in Scheme 4 in the presence of DFcE (aerobic, $x=5, y=50, z=10$ ) at various scan rates: $10,20,50,80,100 \mathrm{mV} \mathrm{s}^{-1}$ from inner to outer. The inset shows the dependence of the cathodic peak current (square) and the assisted proton transfer current at $0.4 \mathrm{~V}$ (triangle) on the square root of the scan rate. 


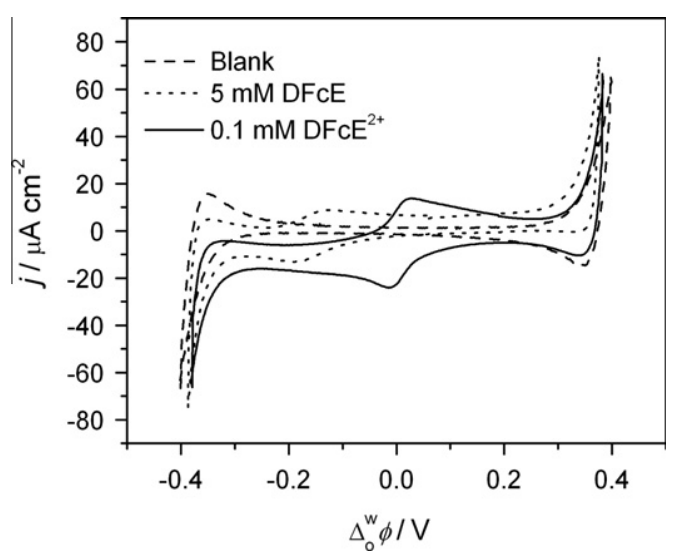

Fig. 9. Comparision of $i R$ compensated voltammograms of $5 \mathrm{mM}$ DFcE (dotted line) and $0.1 \mathrm{mM} \mathrm{DFcE}^{2+}$ (solid line) solutions in the electrochemical cell described in Scheme 4 (aerobic, $y=50, z=0$ ). For comparison, blank CV recorded with $i R$ compensation in the electrochemical cell described in Scheme 4 (dashed line, aerobic, $x=0, y=50, z=0$ ) is also included. Scan rate $=50 \mathrm{mV} \mathrm{s}^{-1}$.

Fig. 9 shows the peaks for reversible transfer of $\mathrm{DFcE}^{+}$(formed after PCET, where the initial species in DCE is DFCE) and $\mathrm{DFCE}^{2+}$ across the $\mathrm{W} / \mathrm{DCE}$ interface at $-0.16 \mathrm{~V}$ and $0.00 \mathrm{~V}$, respectively. $\mathrm{DFcE}^{+}$was formed according to Eqs. (1), (2), as described earlier. The peak separation of the transfer peak of $\mathrm{DFCE}^{2+}$ was about $30 \mathrm{mV}$, confirming that the transferred species had a charge equal to 2. The peak current of $\mathrm{DFcE}^{2+}$ transfer depended on the square root of the scan rate. The Gibbs free energy of transfer for both species was calculated with the following equation.

$\Delta G_{\mathrm{tr}}^{\mathrm{w} \rightarrow \mathrm{o}}=z_{i} F \Delta_{\mathrm{o}}^{\mathrm{w}} \phi^{0} \approx z_{i} F \Delta_{\mathrm{o}}^{\mathrm{w}} \phi^{\prime}$

The transfer energy of the neutral species can be estimated by subtracting the charge dependent part of the transfer energy (Eq. (8)) [42]. This can be calculated for example with the theoretical model of Born (Eq. (9)) or Abraham-Liszi, or with a semiempirical model by Osakai and Ebina [42].

$\Delta G_{\mathrm{tr}, \text { neutral }}^{\mathrm{w} \rightarrow \mathrm{o}}=\Delta G_{\mathrm{tr}, \text { ion }}^{\mathrm{w} \rightarrow \mathrm{o}}-\Delta G_{\mathrm{tr}}^{\mathrm{w} \rightarrow \mathrm{o}}$ (charge dependent)

$\Delta G_{\mathrm{tr}}^{\mathrm{w} \rightarrow \mathrm{o}}$ (charge dependent $)=\frac{N_{A} e^{2}}{8 \pi \varepsilon_{0} r}\left(\frac{1}{\varepsilon_{0}}-\frac{1}{\varepsilon_{\mathrm{w}}}\right)$

where $N_{A}$ is Avogadro's constant, $e$ is the elementary charge, $\varepsilon_{0}$ is the permittivity of vacuum, and $\varepsilon_{\mathrm{w}}$ and $\varepsilon_{\mathrm{o}}$ are the relative permittivity of aqueous and oil phase, respectively taken as 78.54 [42] and 10.42 [43]. The radius of 1,2-diferrocenylethane was estimated to be close to $10 \AA$ (Crystallographic radius of ferrocene is reported as $3.65 \AA$ [44] and the length of the ethane chain is three times $\mathrm{C}-\mathrm{C}$ bond length of $1.54 \AA$ [45]). Eq. (8) was also used to calculate the transfer energy of the neutral species, and the partition coefficient of DFcE $(\log P)$ between DCE and water was also calculated with the following equation [46].

$\log P=-\frac{\Delta G_{\text {tr, neutral }}^{\mathrm{w} \rightarrow 0}}{2.3 R T}$

The difference of the transfer energies of $\mathrm{DFcE}^{+}$and $\mathrm{DFcE}^{2+}$ was calculated to be $17.3 \mathrm{~kJ} / \mathrm{mol}$, while the measured value was $16.7 \mathrm{~kJ} /$ mol. Log $P$ for DFcE was determined as 3.89 or 3.75 , when the transfer energies measured for $\mathrm{DFcE}^{2+}$ and $\mathrm{DFcE}^{+}$were used, respectively. The values are in reasonable agreement with each other, but it has to be taken into account that Eq. (9) is very sensitive to the molecular radius, and the accurate estimation of that is uncertain.

The measured transfer potentials, redox potentials and diffusion coefficients of all the species are tabulated in Table 1.
Table 1

Diffusion coefficients $(D)$, formal potential of oxidation vs. SHE in DCE $\left(E^{\prime}\right)$, the formal transfer potentials $\left(\Delta_{0}^{\mathrm{w}} \phi^{\prime}\right)$ and Gibbs free energies of transfer from water to DCE $\left(\Delta G_{t r}^{\mathrm{w} \rightarrow 0}\right)$ for all observed species of DFcE.

\begin{tabular}{lllll}
\hline & $\mathrm{D} \times 10^{6}\left(\mathrm{~cm}^{2} \mathrm{~s}^{-1}\right)$ & $\mathrm{E}^{\prime}(\mathrm{V}) v s . \mathrm{SHE}$ & $\Delta_{\mathrm{o}}^{\mathrm{w}} \phi^{\prime}(\mathrm{V})$ & $\Delta G_{\mathrm{tr}}^{\mathrm{w} \rightarrow 0}\left(\mathrm{~kJ} \mathrm{~mol}^{-1}\right)$ \\
\hline $\mathrm{DFcE}$ & 5.8 & 0.565 & - & - \\
$\mathrm{DFcE}^{+}$ & 3.7 & 0.77 & -0.162 & -15.6 \\
$\mathrm{DFcE}^{2+}$ & 3.8 & - & 0.005 & 0.96 \\
\hline
\end{tabular}

\subsection{Mechanism}

The measurements indicate that oxygen reduction by DFcE does not proceed through oxygen molecule sandwiched inside the two protonated iron centers of DFcE, as hypothesized by Fomin [19], as no $\mathrm{DFcE}^{2+}$ is observed in four-electrode cell measurements. Thus it is more likely that the reaction proceeds as proposed by Girault et al. for decamethylferrocene through a delocalized triplet transition state, leading to the formation of $\mathrm{DFcE}^{+}$and a hydrogen peroxyl radical [17]. Hydrogen peroxyl radical will then react fast with DFcE and $\mathrm{H}^{+}$to form $\mathrm{H}_{2} \mathrm{O}_{2}$. Alternatively, the reaction could still proceed through molecular oxygen located between two DFcE- $\mathrm{H}^{+}$molecules, but this would require a trimolecular reaction deeming it more unlikely. Another interesting question is, whether the electron is donated by the protonated iron or the non-protonated one. In this case, probably the protonation will take place outside of the molecule due to the steric hindrance of the other ferrocenyl group, and thus the non-protonated iron would be too far to have an effect on the reaction.

Hydrogen peroxide reduction by ferrocene derivatives has been briefly mentioned previously in the literature to explain observed four-electron reduction of molecular oxygen but it has been less well studied $[18,26]$. A mechanism for ferrocene oxidation by hydrogen peroxide suggested by Fomin indicates that the protonated ferrocene can react with $\mathrm{H}_{2} \mathrm{O}_{2}$ to form water, $\mathrm{Fc}^{+}$and $\mathrm{OH}^{\cdot}$ radical, which further reacts with $\mathrm{Fc}$ and a proton to produce water [19]. From this point of view, DFcE seems ideal for hydrogen peroxide reduction, as hydrogen peroxide can react with the protonated ferrocenyl group and the generated $\mathrm{OH}^{*}$ radical can then easily oxidize the other ferrocenyl group. The experimental results show that hydrogen peroxide reduction is faster than oxygen reduction, corroborating the proposed mechanism.

Taking into account all the previous results, it can be proposed that $\mathrm{O}_{2}$ reduction by DFcE can take place at the W/DCE interface or in the bulk DCE phase (see Scheme 5B). The first step consists of the protonation of DFcE to form the DFcE- $\mathrm{H}^{+}$in DCE phase, as observed with other metallocenes. Then, the formed $\mathrm{DFcE}-\mathrm{H}^{+}$is attacked by dissolved $\mathrm{O}_{2}$ in DCE side at the W/DCE interface or in the DCE bulk to produce $\mathrm{H}_{2} \mathrm{O}_{2}$ or water and $\mathrm{DFCE}^{+}$. Hydrogen peroxide reduction is assumed to proceed in a similar manner, but the reaction is assumed to take place close to the interface due to the small solubility of $\mathrm{H}_{2} \mathrm{O}_{2}$ in DCE.

DFcE can also partition into aqueous phase and react with aqueous oxygen or hydrogen peroxide (see Scheme $5 \mathrm{~A}$ ), as shown by $\mathrm{H}_{2} \mathrm{O}_{2}$ decomposition experiments. In that case no protons were present in DCE, so the observed $\mathrm{DFcE}^{+}$in aqueous phase was the reaction product of $\mathrm{H}_{2} \mathrm{O}_{2}$ reduction by partitioned DFCE. In biphasic shake-flask experiments, the protons are extracted to the oil phase by $\mathrm{TB}^{-}$. This fast extraction is followed by slower oxygen reduction initially by $\mathrm{DFcE}$ and later by $\mathrm{DFcE}^{+}$in the bulk DCE phase (see Scheme 5C). From voltammetry and shake-flask results, it can be concluded that the reaction is controlled by the Galvani potential difference applied at the ITIES, which mainly functions as the driving force for the proton pump at the soft molecular interface. 


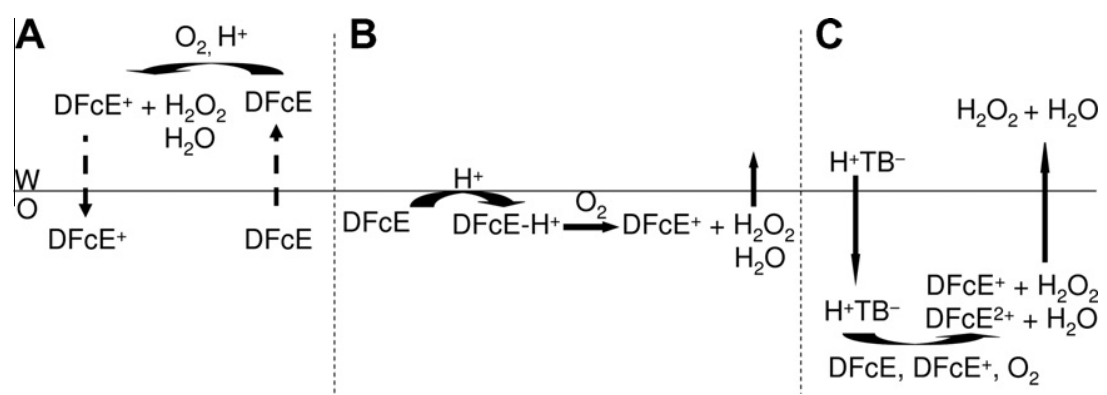

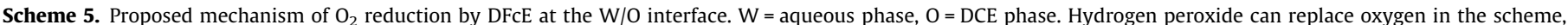

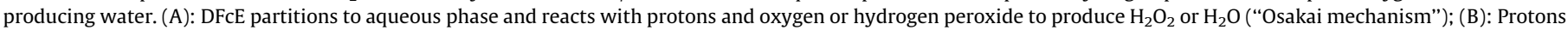

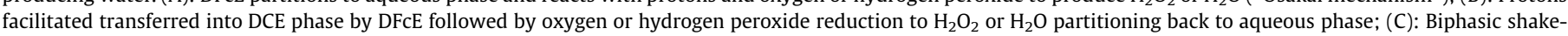
flask reaction where $\mathrm{TB}^{-}$extracts protons to DCE phase, followed by oxygen and hydrogen peroxide reduction in the bulk of DCE phase.

\section{Conclusions}

In summary, we have shown that $\mathrm{O}_{2}$ and $\mathrm{H}_{2} \mathrm{O}_{2}$ reduction by DFcE occurs at a polarized water/DCE interface. The first step consists of the protonation of DFcE to form the DFCE- $\mathrm{H}^{+}$in DCE phase, either by DFcE facilitated proton transfer observed in the fourelectrode cell experiments or by the homogeneous protonation of DFcE in the presence of protons extracted in the oil phase by $\mathrm{TB}^{-}$. The formed DFcE- $\mathrm{H}^{+}$is then attacked by dissolved $\mathrm{O}_{2}$ in DCE side at the W/DCE interface or in the DCE bulk to produce $\mathrm{H}_{2} \mathrm{O}_{2}$ and $\mathrm{DFCE}^{+}$. Hydrogen peroxide is reduced by the protonated DFcE in a similar manner. The products, $\mathrm{H}_{2} \mathrm{O}_{2}$ or $\mathrm{H}_{2} \mathrm{O}$, generated in DCE phase are extracted into the aqueous phase. This separation of products from reactants is one advantage of the biphasic system used herein. The reaction can be turned on by driving protons to the oil phase with the Galvani potential difference across the interface, which can be easily tuned by the chemical way (common ion distribution) or by the external potential polarization. The results show that DFcE can be used as an electron donor for oxygen and hydrogen peroxide reduction, although the reaction without a catalyst is rather slow. This is mainly due to a very low electrochemical driving force, and in the future this type of linked ferrocene compounds could prove to be very interesting electron donors for oxygen reduction and hydrogen evolution, if the redox potential of the compound could be reduced for example by methylation.

\section{Acknowledgments}

The authors thank the China Scholarship Council (CSC) and the Academy of Finland (Grant No. 133261) for financial support. PP also wishes to acknowledge Magnus Ehrnrooth foundation for a travel grant.

\section{References}

[1] H.H. Girault, D.J. Schiffrin, in: A.J. Bard (Ed.), Electroanalytical Chemistry. A series of advances, vol. 15, Marcel Dekker, New York, 1989, p. 1.

[2] H. Ohde, K. Maeda, Y. Yoshida, S. Kihara, J. Electroanal. Chem. 483 (2000) 108

[3] F. Li, B. Su, F.C. Salazar, R.P. Nia, H.H. Girault, Electrochem. Commun. 11 (2009) 473.

[4] B. Su, R. Partovi-Nia, F. Li, M. Hojeij, M. Prudent, C. Corminboeuf, Z. Samec, H.H. Girault, Angew. Chem., Int. Ed. 47 (2008) 4675.

[5] A.J. Olaya, P. Ge, J.F. Gonthier, P. Pechy, C. Corminboeuf, H.H. Girault, J. Am. Chem. Soc. 133 (2011) 12115.

[6] I. Hatay, B. Su, F. Li, M.A. Méndez, T. Khoury, C.P. Gros, J.M. Barbe, M. Ersoz, Z Samec, H.H. Girault, J. Am. Chem. Soc. 131 (2009) 13453.

[7] R. Partovi-Nia, B. Su, F. Li, C.P. Gros, J.M. Barbe, Z. Samec, H.H. Girault, Chem.Eur. J. 15 (2009) 2335

[8] B. Su, I. Hatay, A. Trojánek, Z. Samec, T. Khoury, C.P. Gros, J.M. Barbe, A. Daina, P.A. Carrupt, H.H. Girault, J. Am. Chem. Soc. 132 (2010) 2655.

[9] A. Trojánek, J. Langmaier, B. Su, H.H. Girault, Z. Samec, Electrochem. Commun. 11 (2009) 1940
[10] I. Hatay, B. Su, M.A. Méndez, C. Corminboeuf, T. Khoury, C.P. Gros, M. Bourdillon, M. Meyer, J.M. Barbe, M. Ersoz, S. Záliŝ, Z. Samec, H.H. Girault, J. Am. Chem. Soc. 132 (2010) 13733.

[11] R. Partovi-Nia, B. Su, M.A. Méndez, B. Habermeyer, C.P. Gros, J.M. Barbe, Z. Samec, H.H. Girault, Chem. Phys. Chem. 11 (2010) 2979.

[12] B. Su, I. Hatay, F. Li, R. Partovi-Nia, M.A. Méndez, Z. Samec, M. Ersoz, H.H. Girault, J. Electroanal. Chem. 639 (2010) 102.

[13] P. Peljo, T. Rauhala, L. Murtomäki, T. Kallio, K. Kontturi, Int. J. Hydrogen Energy 36 (2011) 10033.

[14] A.J. Olaya, D. Schaming, P.-F. Brevet, H. Nagatani, T. Zimmermann, J. Vanicek, H.-J. Xu, C.P. Gros, J.-M. Barbe, H.H. Girault, J. Am. Chem. Soc. 134 (2012) 498.

[15] P. Peljo, L. Murtomäki, T. Kallio, H.-J. Xu, M. Meyer, C.P. Gros, J.M. Barbe, H.H. Girault, K. Laasonen, K. Kontturi, J. Am. Chem. Soc. 134 (2012) 5974.

[16] P.W. Atkins, Physical Chemistry, 4th ed., Oxford University Press, Oxford, 1990.

[17] B. Su, I. Hatay, P.Y. Ge, M. Mendez, C. Corminboeuf, Z. Samec, M. Ersoz, H.H. Girault, Chem. Commun. 46 (2010) 2918.

[18] T.E. Bitterwolf, A.C. Ling, J. Organomet. Chem. 40 (1972) C29.

[19] V. Fomin, Russ. J. Gen. Chem. 77 (2007) 954.

[20] C.J. Chang, Z.H. Loh, C. Shi, F.C. Anson, D.G. Nocera, J. Am. Chem. Soc. 126 (2004) 10013.

[21] J.P. Collman, P. Denisevich, Y. Konai, M. Marrocco, C. Koval, F.C. Anson, J. Am. Chem. Soc. 102 (1980) 6027.

[22] P.E.M. Siegbahn, M.R.A. Blomberg, Chem. Rev. 110 (2010) 7040.

[23] N. Eugster, D.J. Fermín, H.H. Girault, J. Phys, Chem. B 106 (2002) 3428.

[24] D.J. Fermín, H.D. Duong, Z. Ding, P.F. Brevet, H.H. Girault, Phys. Chem. Chem. Phys. 1 (1999) 1461.

[25] T.E. Bitterwolf, A.C. Ling, J. Organomet. Chem. 57 (1973) C15.

[26] J. Lubach, W. Drenth, Rec. Trau. Chim. Pays-Bas 92 (1973) 586.

[27] R.M. Sellers, The Analyst 105 (1980) 950.

[28] J.J. Nieminen, I. Hatay, P. Ge, M.A. Méndez, L. Murtomäki, H.H. Girault, Chem. Commun. 47 (2011) 5548.

[29] F. Gao, Y. Yang, J. Liu, H. Shao, Ionics 16 (2010) 45.

[30] T. Wandlowski, V. Mareček, Z. Samec, Electrochim. Acta 35 (1990) 1173.

[31] N. Camire, U.T. Mueller-Westerhoff, W.E. Geiger, J. Organomet. Chem. 637-639 (2001) 823.

[32] J. Xu, A. Frcic, J.A.C. Clyburne, R.A. Gossage, H.Z. Yu, J. Phys. Chem. B 108 (2004) 5742 .

[33] D. Xiang, N. Merbouh, H. Shao, H.Z. Yu, Electrochim. Acta 56 (2011) 5788.

[34] W.E. Geiger, F. Barrière, Acc. Chem. Res. 43 (2010) 1030.

[35] A.M. Nowicka, Z. Stojek, M. Ciszkowska, Anal. Lett. 37 (2004) 979

[36] I. Hatay, B. Su, F. Li, R. Partovi-Nia, H. Vrubel, X. Hu, M. Ersoz, H.H. Girault, Angew. Chem., Int. Ed. 48 (2009) 5139.

[37] S. Fukuzumi, K. Okamoto, C.P. Gros, R. Guilard, J. Am. Chem. Soc. 126 (2004) 10441.

[38] Z. Halime, H. Kotani, Y. Li, S. Fukuzumi, K.D. Karlin, Proc. Natl. Acad. Sci. 108 (2011) 13990.

[39] S. Fukuzumi, H. Kotani, H.R. Lucas, K. Doi, T. Suenobu, R.L. Peterson, K.D. Karlin, J. Am. Chem. Soc. 132 (2010) 6874.

[40] D. Homolka, L.Q. Hung, A. Hofmanova, M.W. Khalil, J. Koryta, V. Marecek, Z. Samec, S.K. Sen, P. Vanysek, Anal. Chem. 52 (1980) 1606.

[41] H. Hotta, S. Ichikawa, T. Sugihara, T. Osakai, J. Phys. Chem. B 107 (2003) 9717.

[42] T. Osakai, K. Ebina, in: A. Volkov (Ed.), Liquid Interfaces in Chemical, Biological and Pharmaceutical Applications, vol. 95, Marcel Dekker, New York, 2001, p. 27.

[43] D.R. Lide (Ed.), CRC Handbook of Chemistry and Physics, 85th ed., CRC Press, Boca Raton, 2005.

[44] N.G. Tsierkezos, U. Ritter, J. Appl. Electrochem. 40 (2010) 409

[45] J. Clayden, N. Greeves, S. Warren, P. Wothers, Organic Chemistry, 1st ed., Oxford University Press, Oxford, 2000.

[46] K. Kontturi, L. Murtomaki, J. Pharm. Sci. 81 (1992) 970. 